\title{
The phantom illumination illusion
}

\author{
DANIELE ZAVAGNO \\ Nagoya University, Nagoya, Japan
}

\begin{abstract}
A novel brightness illusion in planar patterns is reported. The illusion occurs, for example, when surfaces with a luminance ramp shaded from black to white are positioned on a black homogeneous background, so that each white end of the surfaces faces a single point of the plane of the pattern. The illusion consists of the enhancement of the brightness of the background in a relatively wide area around the white ends of the surfaces. A parametric study was conducted in which participants were asked to rate the difference in brightness between the parts of the background inside and outside a virtual circle formed by disks with different luminance ramps. The results show that mean ratings of brightness depended on the luminance of the background, the luminance range of ramps, and the kind of ramp. Discussion of these results with reference to other brightness illusions (assimilation, neon color spreading, anomalous surfaces, visual phantoms, grating induction, and the glare effect) shows that the phantom illumination illusion derives from processes producing the perception of ambient illumination.
\end{abstract}

In this article, I present the phantom illumination (PI) illusion, a new brightness effect occurring in planar patterns. Figure 1 shows examples of the illusion. In Figure $1 \mathrm{~A}$, five disks shaded from black to white form a virtual pentagon on a homogeneous black background, with the white end of each luminance ramp facing the center of the pentagon. During an informal interview, about 25 naive observers reported that the area of the background within the virtual pentagon looked brighter than that in the rest of the background. Some observers reported the impression that the illumination of this area differed from that of the rest of background. No observer reported that the background looked darker than the target area. Since all observers described the illusion as an enhancement of the brightness of the part of the background enclosed by the luminance ramps rather than as a depression of the brightness of the rest of the background, such an enhancement may be considered the main perceptual feature of the illusion.

In general, let us call surfaces with a luminance ramp potentially capable of producing the PI illusion inducers, and let us call any area of the background whose brightness is affected by inducers the target area (T). Figures $1 \mathrm{~B}$ and $1 \mathrm{C}$ show that inducers of different shapes forming different geometric configurations produce the PI illusion when the bright ends of the inducers face a single point of the ideal plane of the pattern. This point is infinitely distant from the inducers in Figure 1B and is

I thank Sergio Cesare Masin for his valuable comments, his editing advice, and his constant support. I also thank Alan Gilchrist, whose lab I used to run the experiment. I dedicate this work to the memory of Prof. Nicholas Pastore, a generous friend and a honest mind. Correspondence concerning this article may be addressed to D. Zavagno, Space Medicine Research Center, Research Institute of Environmental Medicine, Nagoya University, Furo-cho 1, Chikusa-ku, Nagoya 464-8601, Japan (e-mail: dzavagno@danzava.org). relatively close to the inducers in Figure 1C. Figure 1D, in which the man and the child are on a dark background with uniform luminance, shows that the PI illusion may also occur in rather complex visual scenes, thus suggesting that pictorial 3-D information from inducers may be important for the occurrence of the illusion.

In one of its simplest forms, as for example in Figure 1A, the PI illusion recalls other brightness effects. With reference only to stationary achromatic patterns, the following is a list of brightness illusions having some aspect in common with the PI illusion.

Assimilation (Bezold, 1876). Figure 2A provides an example of assimilation (Musatti, 1953). All six backgrounds have the same luminance. When observers look at the patterns in Figure 2A with a global viewing attitude, they may describe each of the three backgrounds on the left as looking lighter than each of the three backgrounds on the right. That is, when the small achromatic disks shown in Figure $2 \mathrm{~A}$ are placed on the respective backgrounds and the observer's viewing attitude is global, the brightness of the backgrounds may be reported to change to a value closer to the brightness of the disks. However, when observers inspect the backgrounds analytically, they often report the reverse, that is, that the three right backgrounds are lighter than the three left backgrounds (Kanizsa, 1979, 1980). As in the assimilation effect, in the PI illusion the brightness of the target area also changes to a value closer to the brightness of the white ends of the inducers. However, the PI illusion differs from the assimilation effect due to the fact that the enhancement of the brightness of the target area persists when the viewing attitude is changed from global to analytical.

Visual phantoms (Gyoba, 1983; Rosenbach, 1902; Tynan \& Sekuler, 1975). When a low-frequency vertical sinusoidal grating of low amplitude (Sakurai \& Gyoba, 1985) or when a square grating (Kitaoka, Gyoba, \& Kawabata, 1999) is occluded by a horizontal black bar, as in Fig- 

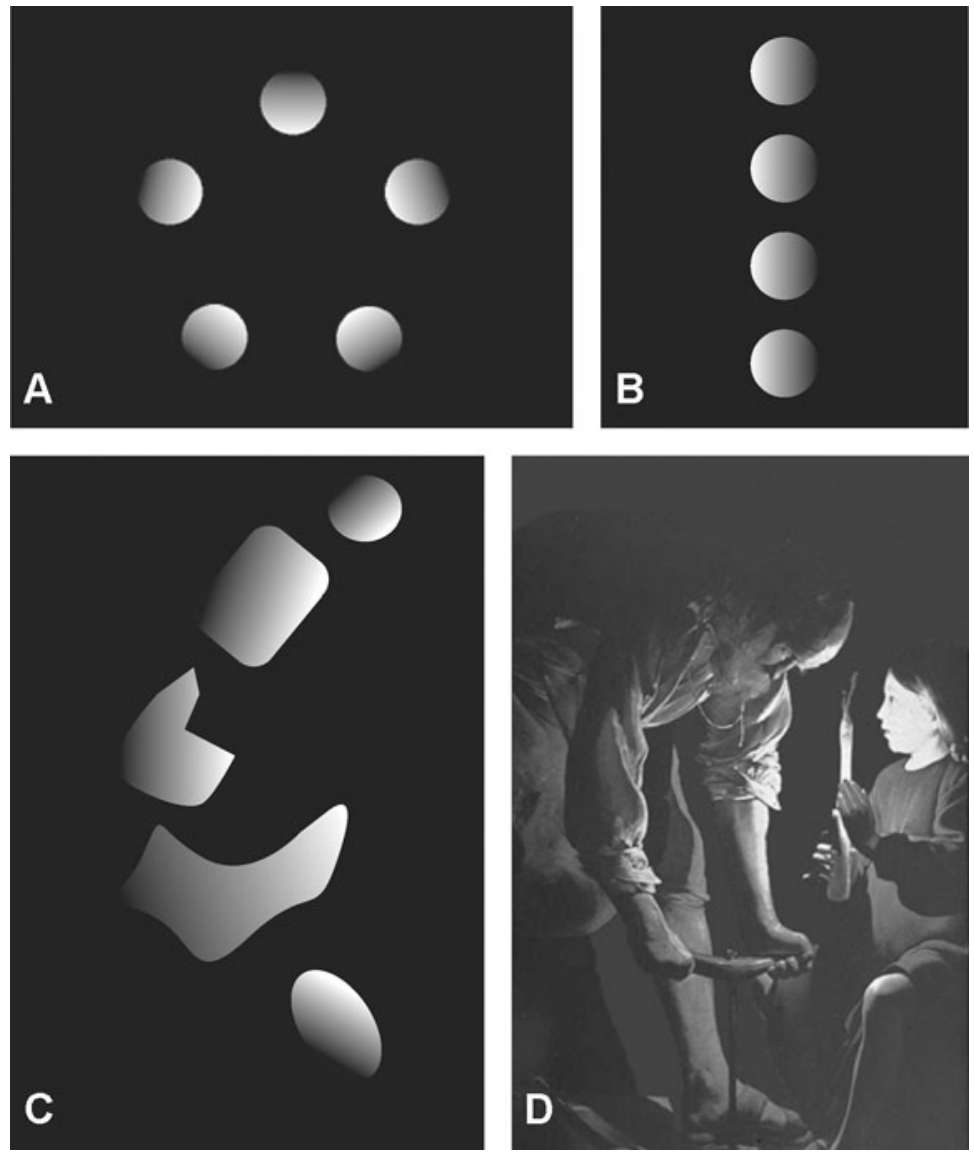

Figure 1. The phantom illumination illusion in four different configurations. (A) Basic configuration used in the parametric study. (B) Configuration that recalls visual phantom patterns. Completion is not required here in order to perceive a background with two different brightness levels. (C) The illusion can be generated with any combination of shapes, as long as the luminance ramps form a coherent luminance polarity chain. (D) Detail from Saint Joseph Carpenter by Georges de La Tour (ca. 1640-1645; Louvre Museum). The background was digitally modified in order to make its reflectance homogeneous. Nevertheless, the luminance ramps depicted by the artist are all that is needed to perceive differences in ambient illumination within the scene. The illusion works even with chromatic displays.

ures $2 \mathrm{~B}$ and $2 \mathrm{C}$, respectively, the grating completes behind the black bar, with this bar appearing transparent. As is exemplified in Figure 2D, when the amplitude of the sinusoidal grating is increased considerably, counterphase brightness inductions (McCourt, 1982, 1994) rather than visual phantoms are perceived. Luminance gradients are a common feature of the PI illusion, of visual phantoms with sinusoidal gratings, and of McCourt's effect. However, the PI illusion differs essentially from visual phantoms and the McCourt effect because its occurrence does not require any completion of occluded patterns. In addition, grating induction appears to affect most of all the edges of the occluder with limited filling in (Figure 2D), whereas the PI illusion appears capable of spreading over larger areas.

Neon color spreading (Van Tuijl, 1975; Varin, 1971). Figure 2E shows a case of achromatic color spreading.
The shade of gray of the phenomenal transparent square is similar to the gray of the parts of the circles overlapped by the square. As in the assimilation effect and the PI illusion, the brightness of the area of the background occupied by the transparent square is closer to that of the circles in this part. Kanizsa (1980) also showed anomalous transparency effects closely related to neon color spreading. However, the PI illusion and neon color spreading are different phenomena because in the PI illusion, one perceives a change in illumination or in brightness of the target area with no segregated surface, but in the area of the background where neon color spreading occurs, one perceives a surface segregated from the background.

Glare effect (Zavagno, 1999; see also Da Pos \& Zambianchi, 1996). Figure 2F shows examples of the glare effect. The white square enclosed within the shaded squares appears more or less luminous depending on 


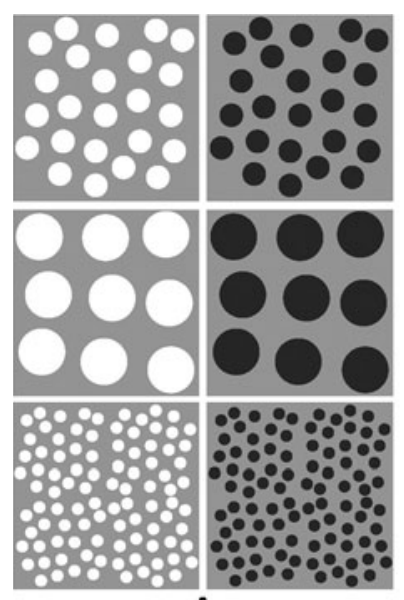

A

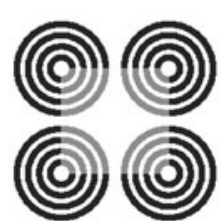

$\mathrm{E}$

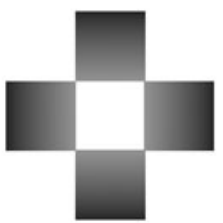

$+2$

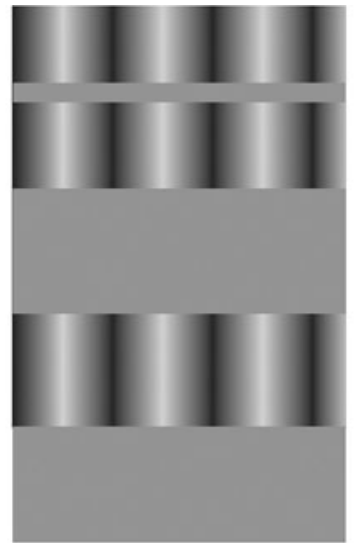

D

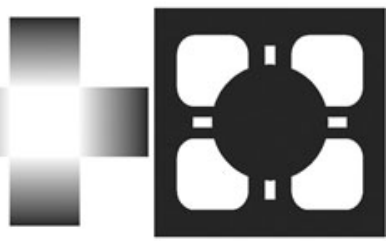

G

Figure 2. (A) Top: Simultaneous assimilation display modified after Musatti (1953). Center and bottom: By increasing or reducing the size of the inducers, one should be able to manipulate the magnitude of the assimilation effect; the differences, however, are rather weak. (B) Scotopic visual phantom illusion (Gyoba, 1983), a completion effect in phase with the low-range and low-frequency sinusoidal grating. (C) Photopic visual phantom illusion (Kitaoka, Gyoba, \& Kawabata, 1999), a completion effect that shows a phantom transparency in phase with the square grating. (D) Grating induction illusion (McCourt, 1982) in which lightness induction is counterphase with respect to the sinusoidal grating. The effect seems to be restricted to the edges of the occluder, with limited filling-in capacity. (E) Neon color spreading, a completion effect that shows an anomalous transparent figure. By covering all of the black sectors of the rings, one will see an ambiguous figure, which might appear as an anomalous transparency similar to the phenomenon described by Kanizsa (1980), or as an anomalous figure, or as a square hole. In all three cases, however, the brightness outcome is opposite to that of neon color spreading. (F) Glare effect with different luminance ramp ranges (modified after Zavagno \& Caputo, in press). (G) One of Kanizsa's anomalous figures; the brightness of the anomalous figure stands in an inverse relationship with the lightness of the inducers.

whether the luminance ramps surrounding it are more or less steep, respectively. Similar configurations may also produce the perception of self-luminous grays (Zavagno $\&$ Caputo, 2001, in press). A similar brightness effect, although somewhat weaker, can be experienced when employing texture gradients (Kennedy, 1976; Zavagno \& Massironi, 1997). The enhancement of the brightness of the target area is a common feature of the PI illusion and the glare effect. However, the PI illusion differs essentially from the glare effect because the latter effect involves processes producing the impression of emission of light from the target area, but the PI illusion involves processes producing the impression of differential lighting of the target area.

Anomalous surfaces (Kanizsa, 1955). Figure 2G shows an example of an anomalous surface; the black disk in the center of the pattern is perceived as a surface segregated from the background. The contour of this phenomenal surface corresponds to no luminance contrast with the black background. Some observers have noted that the PI illusion involves a sort of tenuous contour similar to that of anomalous surfaces. However, the PI illusion differs from anomalous surfaces in that the white ends of inducers in the PI illusion increase the brightness of the target area, but the white inducers of the anomalous surface decrease the brightness of the target area. Presumably, anomalous surfaces and neon color spreading surfaces are produced by the same or similar processes. The PI illusion must depend on a different mechanism, because it does not involve the generation of any illusory surface.

In the present experiment, a parametric study on the PI illusion was conducted to verify the roles of both luminance ramps and the luminance of the background in generating a brightness enhancement in the target area. For this purpose, the geometric layout of Figure 1A was chosen for all stimuli. The independent variables were the luminance of the background and the photometric 
properties of luminance ramps. Features of luminance ramps such as luminance range, contrast polarity with the background, and type of luminance transition (smooth or abrupt) were manipulated. The determination of how these independent variables affected the brightness of the target area is important because this analysis should allow confirmation of whether or not the PI illusion is reducible to some of the other brightness illusions mentioned above.

\section{METHOD}

\section{Participants}

The participants were 22 undergraduate and graduate students from Rutgers University, Newark campus (13 female and 9 male, with an average age of 23). All participants had normal or corrected-tonormal visual acuity and were naive with respect to the purpose of the experiment.

\section{Material}

In a dimly lit room, each stimulus was shown in the middle of the 17-in. display screen of an Apple Multiscan computer with resolu- tion set at $800 \times 600$ pixels. Figure 3 shows the stimuli that were presented using SuperLab. The background covered the entire display screen. The diameter of the disks was $2.5 \mathrm{~cm}$, and the disks were arranged around an imaginary circle with a diameter of $7.3 \mathrm{~cm}$ passing through the center of the disks. The viewing distance was $115 \mathrm{~cm}$.

Stimuli and their background were achromatic. The luminances $1.8,17.3$, and $65 \mathrm{~cd} / \mathrm{m}^{2}$ were used, which corresponded respectively to perceptions of black, gray, and white. For the sake of simplicity, stimuli are described only in terms of these colors. A 3 (background) $\times 2$ (kind of disk) $\times 9$ (color combination) factorial design was used. The backgrounds were black (B), gray (G), or white (W). Disks were either split in two halves (s, split disks) or had a luminance ramp (r, ramp disks); for split disks, each half of the disk had a uniform luminance, but for ramp disks, luminance varied linearly with distance along the diameter of the disk from one to the other of two luminances taken from the above set of three. The color combinations for split disks and the extreme ends of ramp disks were the following: $\mathrm{b}$ (solid black), g (solid gray), w (solid white), b-g (blackgray), b-w (black-white), g-b (gray-black), g-w (gray-white), w-b (white-black), w-g (white-gray). Only 42 of the resulting 54 combinations were used, since the remaining 12 corresponded to empty
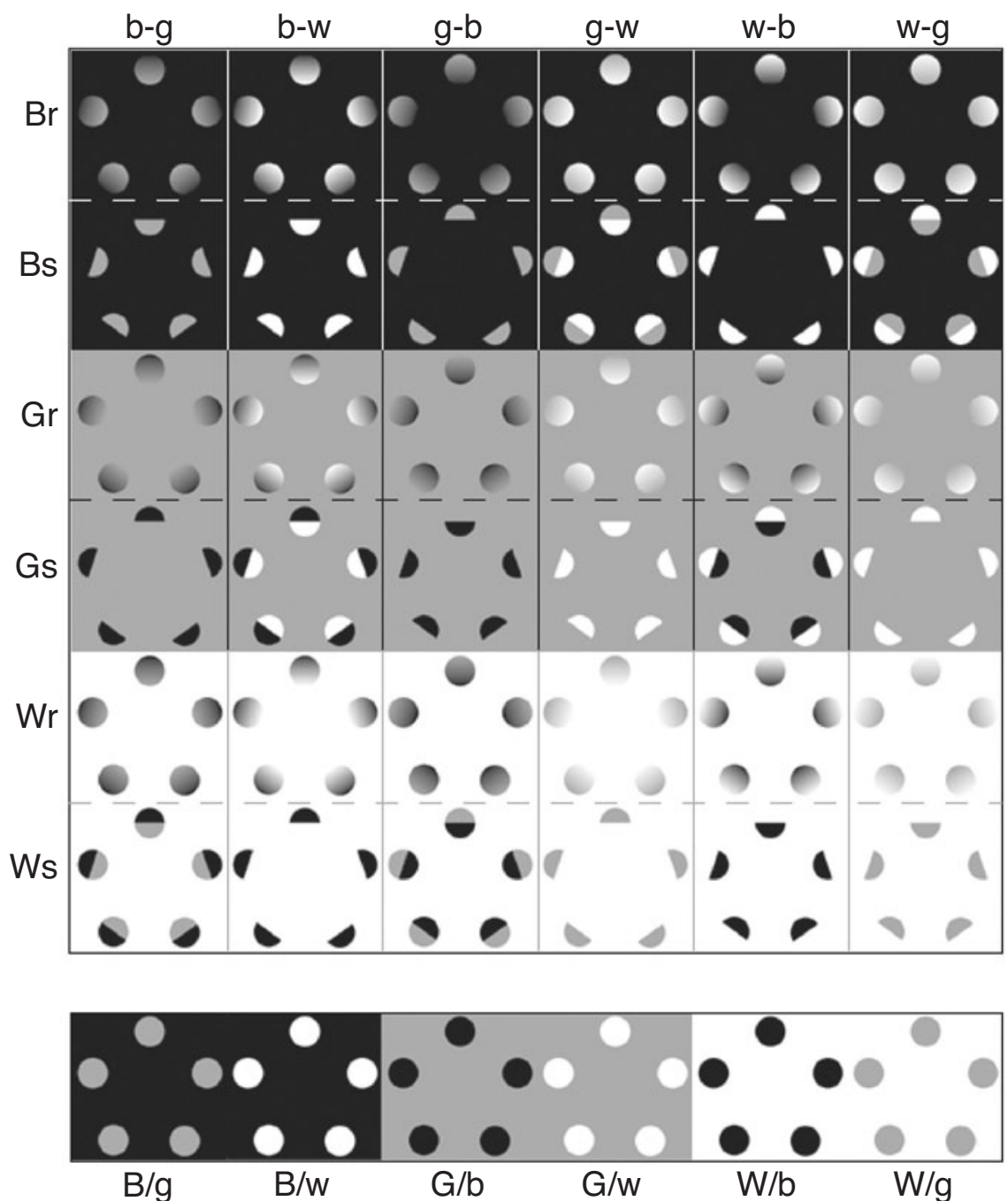

Figure 3. All of the configurations used in the experiment. See text for the meaning of the notation. 
backgrounds or replications of solid-disk stimuli. For ramp disks, I shall distinguish between positive ramps - for example, the stimulus pattern $\mathrm{Br} / \mathrm{b}$-g in Figure 3, in which the brightest ends of the luminance ramps face inward - and negative ramps - for example, the stimulus pattern Br/g-b in Figure 3, in which the brightest ends of the luminance ramps face outward.

Different combinations of factors may involve stimulus patterns that could be considered improper patterns, ${ }^{1}$ in the sense that they generate other brightness effects. For instance, $\mathrm{Wr} / \mathrm{b}-\mathrm{w}$ and $\mathrm{Br} / \mathrm{g}-\mathrm{b}$ generate, respectively, the glare and black hole effects (Zavagno,
1999); Ws/b-g and Gs/g-b generate the neon color spreading effect or anomalous transparencies (Kanizsa, 1980; Van Tuijl, 1975; Varin, 1971); and Gs/b-g and Bs/w-b generate anomalous figures (Kanizsa, 1955, 1979).

\section{Procedure}

Each participant was asked to rate the difference in brightness between $T$, surrounded by the inducers, and the rest of the background using integers from -10 to 10 . Negative values were used when the target area looked darker than the rest of the background,

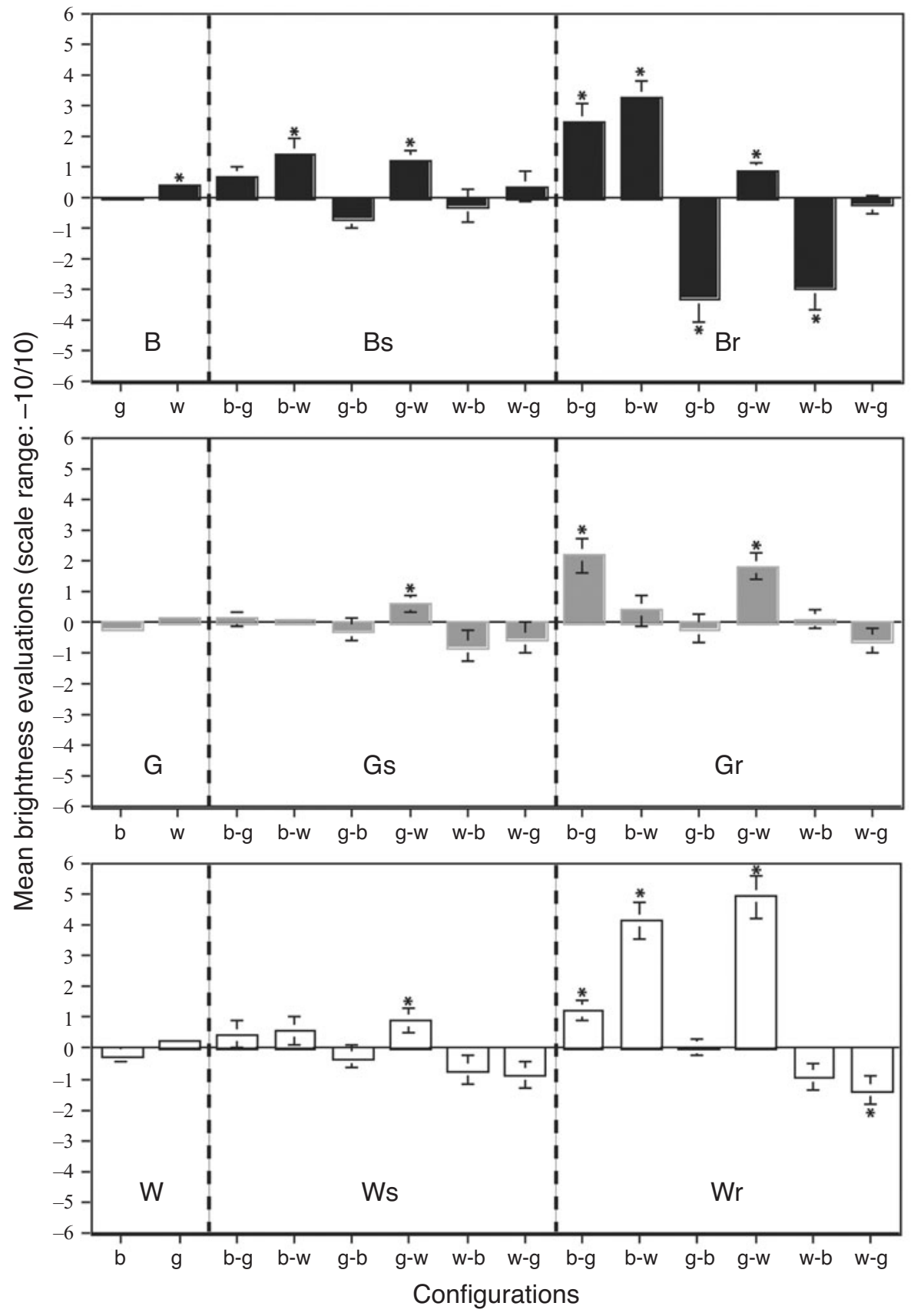

Figure 4. From top to bottom: Mean ratings for the black, gray, and white backgrounds, respectively. Labels on the $x$-axes correspond to the configurations shown in Figure 3 . Error bars are mean standard errors. Asterisks indicate mean evaluations statistically different from 0 (see Table 1). 
positive values when the target area looked lighter than the rest of the background, and " 0 " when the target area was equal in brightness to the rest of the background. Before the actual experiment began, three examples were shown to clarify the use of the scale. The examples consisted of (1) a homogeneous gray background $\left(17.3 \mathrm{~cd} / \mathrm{m}^{2}\right)$ for 0 ; (2) the same background with a brighter disk at the center for a positive rating; and (3) the same background with a dark central disk for a negative rating. The participants were told that those examples were not actual stimuli and that they would not have access to them during the experiment. None of the participants showed difficulties in understanding the task or the use of the scale. Throughout the whole experiment, the first question posed at the appearance of each stimulus was "Does the target look darker, brighter, or the same as the surround?" Responses to this question were collected on a separate sheet to control whether the participants' brightness experiences were consistent with the numerical evaluations they were then asked to provide for the same stimulus. The duration of the experiment ranged from 35 to $60 \mathrm{~min}$. Brief pauses were allowed for rest on request of the participant. Stimuli were presented one at a time twice, in random order.

\section{RESULTS}

For each stimulus, the mean of the two ratings produced by each participant was used as an individual rating. Figure 4 shows mean individual ratings of brightness for each stimulus. Despite the fact that mean ratings ranged from about -4 to +5 , results are consistent in showing both that ramp disks produce overall larger effects in terms of brightness differences between the target and the surround, and that gray backgrounds appear more resistant to brightness changes.

An analysis of variance (ANOVA) showed that the effects of the factors kind of disk $[F(1,21)=18.43, p<$ $.0005]$ and color combination $[F(8,21)=27.307, p<$ $.0001]$ were significant, but the effect of background was not $[F(2,21)=2.360, p=.1]$. This latter finding can be explained by the fact that each background shows the same average pattern of results with respect to split-disk and ramp-disk stimuli. Nevertheless, since data for solid disks were computed twice in order to have a balanced ANOVA between ramp and split disks, and since only configuration B/w (see Figure 4 and Table 1) produced a slight brightness effect, a new ANOVA without data for the single-colored disks was run to check again for a possible significant effect of background luminance. This time the effect of this factor was marginally significant $[F(2,21)=3.145, p=.053]$.

Figure 5 shows the data grouped by color combination without the solid disks. A comparison of the patterns of results for ramp and split disks shows that the differences go beyond the magnitude of the brightness effects for the two classes of stimuli. Of course, this result may in part depend on the fact that some split-disk stimuli show only one half of a disk, as for instance in the case of either $\mathrm{Bs} / \mathrm{b}-\mathrm{w}$ or $\mathrm{Ws} / \mathrm{b}-\mathrm{w}$, in which (respectively) the components " $b$ " and " $w$ " are not seen.

All interactions in each of the ANOVAs were found to be highly significant $(p<.0001)$. This finding is not surprising, since the same combinations of color combination + kind of gradient produced effects of different magnitudes for each background. For instance, the rampdisk color combination $\mathrm{b}-\mathrm{w}$ produced relatively strong effects against both the black and the white backgrounds, but that combination had no significant effect against a gray background. For split disks, $b$-w showed instead a small but significant effect only for the white background (see Figure 5 and Table 1).

One-sample $t$ tests were carried out to verify which configurations received evaluations statistically different from $0(0=$ no difference between target and background), and the results are reported in Table 1.

Table 2 organizes the means for split disks according to what brightness effect they elicited (data for solid disks are excluded). The table also includes the general prediction of what kind of brightness difference should be expected given a specific illusion (anomalous figures, assimilation, etc.) and the polarity of physical contrast between the visible inducer ends and $\mathrm{T}$ (increment or decrement with respect to T). In almost all cases, the direction of the mean brightness difference is consistent with the prediction, even if evaluations reached statistical significance only in 4 cases out of 18 (see also Table 1). Table 3 groups the mean evaluations, respectively for positive and negative ramps; there are eight significant effects with positive ramps but only three with negative ramps.

\section{DISCUSSION AND CONCLUSIONS}

The main findings of the present experiment can be summarized as follows: (1) In the kind of stimulus pattern used in this study, split disks are on average less effective than ramp disks in generating brightness differences on achromatic homogeneous backgrounds (Figures 4 and 5). (2) The differences in ratings suggest that ramp disks induce brightness effects that are intrinsically different from effects such as neon color spreading, anomalous transparencies, and Kanizsa figures.

As noted earlier, split-disk configurations recall certain brightness effects, but at the same time they are "improper" or "not so good" versions of the illusions, such as Kanizsa figures (for example, Bs/g-b; see Figure 3),

Table 1

One-Sample $t$ Tests (Hypothesized Mean $=0$ )

\begin{tabular}{|c|c|c|c|c|c|c|c|c|c|}
\hline & $\mathrm{w}$ & $\mathrm{s} / \mathrm{b}-\mathrm{w}$ & $\mathrm{s} / \mathrm{g}-\mathrm{w}$ & $\mathrm{r} / \mathrm{b}-\mathrm{g}$ & $\mathrm{r} / \mathrm{b}-\mathrm{w}$ & $\mathrm{r} / \mathrm{g}-\mathrm{b}$ & $\mathrm{r} / \mathrm{g}-\mathrm{w}$ & $\mathrm{r} / \mathrm{w}-\mathrm{b}$ & r/w-g \\
\hline $\mathrm{B}$ & $2.4^{*}$ & $2.87^{* *}$ & $3.82^{\dagger \dagger}$ & 4.82 t末 & $5.99 \$$ & $-4.1 \ddagger$ & $3.45^{\dagger}$ & -4.12 & \\
\hline G & & & $2.15^{*}$ & $3.91^{\dagger \dagger}$ & & & $3.97 \dagger$ & & \\
\hline W & & & $2.4^{*}$ & $3.81^{\dagger \dagger}$ & $6.81 \ddagger$ & & $6.9+4$ & & $-2.78^{*}$ \\
\hline
\end{tabular}




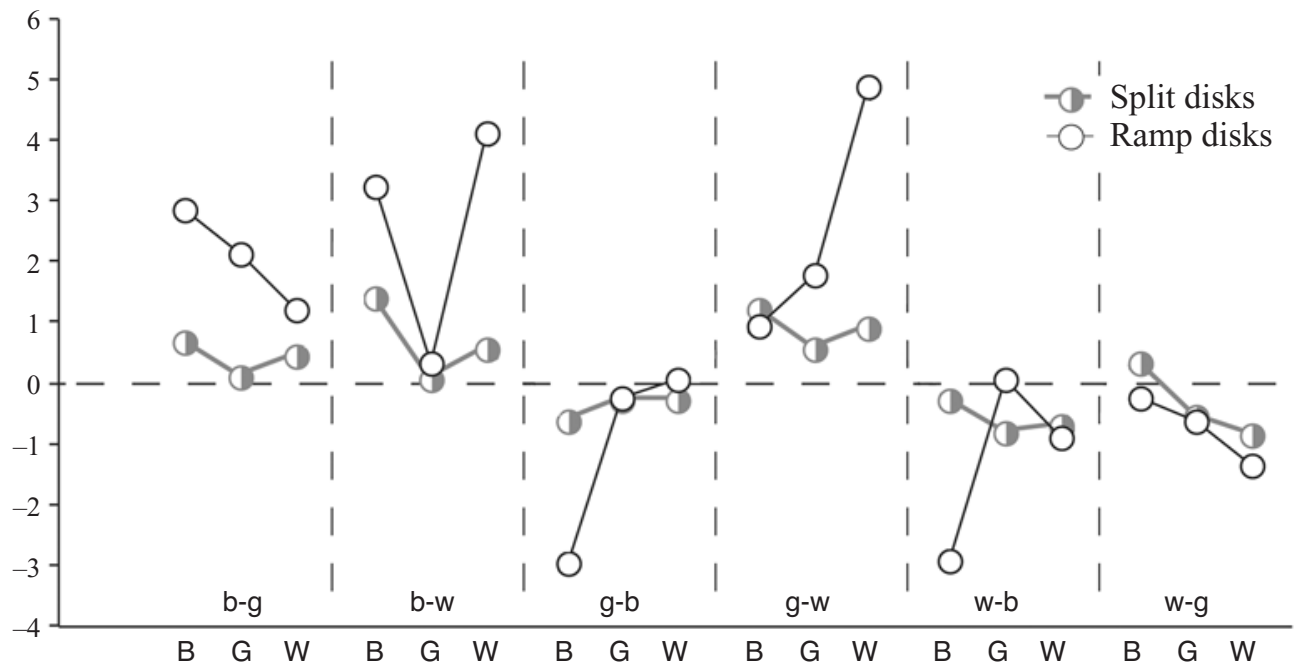

Figure 5. Mean ratings without solid disks, grouped by color combination.

neon color spreading $(\mathrm{Bs} / \mathrm{g}-\mathrm{w})$, or even other anomalous transparency effects (Kanizsa, 1980) (Bs/b-w). Therefore, despite the facts that recent literature has shown that luminance ramps play a special role with color perception (Agostini \& Galmonte, 1997, 2002b; Bressan, 2001; Logvinenko, 1999) and are capable of generating very strong brightness illusions (Leonards, Ibanez, Zavagno, Seghier, \& Troscianko, 2003; Zavagno, 1999), it would be inappropriate in this case to state simply that brightness effects caused by luminance ramps are stronger overall than classical brightness effects without luminance ramps. The point in question, however, was neither a measurement of the magnitude of those effects nor a direct comparison between magnitudes. Instead, one of the goals of this work was to understand whether the phantom illumination illusion could be reduced to another brightness effect. This does not seem to be the case with reference to brightness effects related to the formation of phenomenal surfaces (neon color spreading, Kanizsa figures, and transparencies). Strong figural constraints that involve concurring gestalt organization principles (Kanizsa, 1979; Metzger, 1971; Wertheimer, 1923) are in fact required in order to experience such effects. In the experiment reported, those constraints were somewhat weakened (see note 1), which might be one of the reasons for the shallow range of evaluations associated with split-disk configurations. The PI illusion instead appears to have fewer, or at least different, configuration constraints (Figure 1). This situation holds true also for the glare effect, which is probably one of the reasons why ramp disks received in absolute terms higher ratings than patterns without luminance ramps, even when the ramp-disk configurations used-just as in the case of split disks - were not the best ones to show those effects (Zavagno, 2001). Yet, the difference in the absolute magnitudes of effects between split-disk and ramp-disk stimuli strongly suggests two distinct classes of effects.

The PI illusion and the glare effect thus appear to be closely related. But can the PI illusion be accounted for by using the same principles that appear to govern the glare effect (Zavagno \& Caputo, 2001)? The present ex-

Table 2

Split-Disk Stimuli and Respective Means Grouped by Type of Phenomenal Experience They May Generate

\begin{tabular}{|c|c|c|c|c|}
\hline \multirow[b]{2}{*}{ Phenomenal Experience } & \multicolumn{2}{|c|}{ Increment for $\mathrm{T}$} & \multicolumn{2}{|c|}{ Decrement for $\mathrm{T}$} \\
\hline & Stimulus & $M$ & Stimulus & $M$ \\
\hline \multirow[t]{3}{*}{ Anomalous transparency (in-phase) } & $\mathrm{Bs} / \mathrm{b}-\mathrm{g}$ & 0.66 & Gs/g-b & -0.27 \\
\hline & Bs/b-w & $1.4^{\mathrm{a}}$ & Ws/w-b & -0.7 \\
\hline & Gs/g-w & $0.57^{\mathrm{a}}$ & Ws/w-g & -0.84 \\
\hline \multirow[t]{3}{*}{ Kanizsa figure (counterphase) } & $\mathrm{Bs} / \mathrm{g}-\mathrm{b}$ & -0.64 & Gs/b-g & 0.09 \\
\hline & $\mathrm{Bs} / \mathrm{w}-\mathrm{b}$ & -0.27 & $\mathrm{Ws} / \mathrm{b}-\mathrm{w}$ & 0.54 \\
\hline & Gs/w-g & -0.54 & Ws/g-w & $0.89^{\mathrm{a}}$ \\
\hline Neon color spreading (in-phase); & $\mathrm{Bs} / \mathrm{g}-\mathrm{w}$ & $1.2^{\mathrm{a}}$ & $\mathrm{Gs} / \mathrm{w}-\mathrm{b}$ & -0.82 \\
\hline Assimilation (in-phase); Holes (no & $\mathrm{Bs} / \mathrm{w}-\mathrm{g}$ & 0.34 & Ws/b-g & 0.45 \\
\hline difference) & $\mathrm{Gs} / \mathrm{b}-\mathrm{w}$ & 0.04 & Ws/g-b & -0.27 \\
\hline
\end{tabular}

aMean is statistically different from 0 (see Table 1). 
Table 3

Ramp-Disk Configurations and Respective Means

\begin{tabular}{cccccccc}
\hline & \multicolumn{3}{c}{ Negative Ramps } & & \multicolumn{3}{c}{ Positive Ramps } \\
\cline { 2 - 4 } \cline { 7 - 8 } Background & $\mathrm{g}-\mathrm{b}$ & $\mathrm{w}-\mathrm{b}$ & $\mathrm{w}-\mathrm{g}$ & & $\mathrm{b}-\mathrm{g}$ & $\mathrm{b}-\mathrm{w}$ & $\mathrm{g}-\mathrm{w}$ \\
\hline Black & $-3.25^{\mathrm{a}}$ & $-2.9^{\mathrm{a}}$ & -0.23 & & $2.84^{\mathrm{a}}$ & $3.23^{\mathrm{a}}$ & $0.89^{\mathrm{a}}$ \\
Gray & -0.25 & 0.04 & -0.64 & & $2.14^{\mathrm{a}}$ & 0.34 & $1.79^{\mathrm{a}}$ \\
White & -0.04 & -0.9 & $-1.34^{\mathrm{a}}$ & & $1.23^{\mathrm{a}}$ & $4.13^{\mathrm{a}}$ & $4.89^{\mathrm{a}}$ \\
\hline
\end{tabular}

aMean is statistically different from 0 (see Table 1 ).

periment cannot offer a final answer to this question, but it can inform us about substantial differences and similarities between the two illusions.

For instance, leaving aside for the moment the results for gray backgrounds, a common principle seems to fit the data for black and white backgrounds with ramp disks: Positive ramps induce brightening effects, and negative ramps induce darkening effects (see Table 3 ). This principle works regardless of whether the inner end of a ramp is an increment or a decrement with respect to $\mathrm{T}$. For instance, the ramp-disk color combination b-g is a positive ramp for both black and white backgrounds, whereas $w-b$ is a negative ramp for those backgrounds.

The aforementioned principle states the direction of the brightness evaluation of $\mathrm{T}$ with respect to the surround $(\mathrm{S})$, but it does not inform us about anything else. To understand whether the two illusions can be accounted for by the same mechanisms, one needs also to understand what variables affect the magnitude of brightness changes. In this regard, Zavagno \& Caputo (2001) observed that the luminance range of the ramps is critical in the appreciation of the glare effect (see Figure 2F). Given such evidence, one might think that the luminance range of ramps also has a major effect on the brightness differences between $\mathrm{S}$ and $\mathrm{T}$ in the case of the PI illusion. A full-range ramp (in our case $b-w$ or $w-b$, expressed by a ratio of more than 30:1 between the bright and dark ramp ends) should generate greater effects than smaller-range ramps (about 4:1 for g-w and w-g, and about 10:1 for b-g and g-b). As one can see from Figures 4 and 5, however, this principle does not apply in a linear way to the black and white backgrounds. For instance, $\mathrm{Br} / \mathrm{b}$-g and $\mathrm{Wr} / \mathrm{b}$-g show strong differences in in their mean ratings, while $\mathrm{Br} / \mathrm{b}-\mathrm{g}$ and $\mathrm{Br} / \mathrm{b}-\mathrm{w}$ received instead similar brightness evaluations despite the big differences in the luminance range of their ramps.

Nevertheless, the difference in evaluations between $\mathrm{Br} / \mathrm{b}-\mathrm{g}$ and $\mathrm{Wr} / \mathrm{b}-\mathrm{g}$ might reflect something else going on. A closer look at the data, in fact, reveals that the brightening or darkening of $\mathrm{T}$ is improved when the physical contrast between $T$ and the inner ends of ramps is null. If this holds true for the inner ends, why should it not also work for the outer ends with respect to S? In other words, the brightness appearance of T might depend to some extent on the effect exerted by the outer ends of the gradient on $\mathrm{S}$. This would explain why $\mathrm{Br} / \mathrm{b}$-g appears brighter than $\mathrm{Wr} / \mathrm{b}$-g, given that the ramp b-g would have a major darkening effect on the black surround.
On the other hand, one must also consider that a strong asymmetry exists between brightening and darkening effects, in that the latter are much less conspicuous. This fact is particularly evident in a comparison of the brightening effect with $\mathrm{Br} / \mathrm{b}-\mathrm{w}$ and the darkening effect with $\mathrm{Wr} / \mathrm{w}-\mathrm{b}$ : If the outer ends played a major role in affecting the appearance of $\mathrm{T}$ (by affecting the appearance of $\mathrm{S}$ ), then one would expect the absolute magnitude of $\mathrm{Wr} / \mathrm{w}-\mathrm{b}$ to be equal to, if not greater than, the absolute magnitude of $\mathrm{Br} / \mathrm{b}-\mathrm{w}$. In fact, with the $\mathrm{Wr} / \mathrm{w}-\mathrm{b}$ pattern (1) w-b is a full-range ramp; (2) the ramp is positive with respect to $S$ (and thus should brighten up S); and (3) the ratio between $\mathrm{S}$ and the outer end is null. All of these conditions should favor a background glare effect. However, the evaluation of $\mathrm{T}$ for $\mathrm{Wr} / \mathrm{w}-\mathrm{b}$ is not statistically different from 0 (Table 3).

If we add to these observations also the data for gray backgrounds, we find a nonlinearity in the evaluations for positive ramps that adds to the strong asymmetry between the effects of the positive and negative ramps described above.

Therefore, even if the present experiment cannot offer the final word about the psychological mechanisms governing the PI illusion and the glare effect, a closer look at the data collected does suggest that the two illusions obey different rules. This fact is somewhat reflected also by the phenomenal experience related to the two illusions. In the case of the glare effect and its opposite, the black hole effect, both are experienced as if they were inherent qualities of the target itself. In the case of the PI illusion and its opposite, however, the brightness effects are experienced as if they depend upon something else, an external agent: They are not seen as inherent properties of the target. Still, in both cases it is clear that luminance ramps are information used by the system to process brightness. In that sense, luminance ramps appear to be specific information both for luminosity and illumination perception (Zavagno \& Caputo, 2001, in press). As this experiment shows, however, the outcome of one experience but not the other is clearly dependent upon specific luminance interactions between the target, the inducers, and the background.

One last question is left to consider: Does the PI illusion fit within the category of assimilation? As stated in the introduction, the direction of the effect appears to comply with assimilation. From classic studies on assimilation (Burnham, 1953; Helson \& Rohles, 1959; Newhall, 1942), it appears that the magnitude of brightness assimi- 
lation depends on the ratio of the metric dimensions of inducing elements and their number: By increasing the dimensions of inducers while decreasing their number by some proportion, assimilation should be gradually reduced, and vice versa. If we consider, for example, the displays in Figure 2A (modified after Musatti, 1953), we will notice that the inducers are randomly placed all over the gray surfaces. If the size of the inducers is increased (Figure 2A center), the effect should become less conspicuous, maybe even reverting into contrast. If the sizes are instead reduced and the numbers of inducers are increased (Figure 2A bottom), assimilation should become stronger. Even though we do not know at this stage whether similar ratios hold true also for the PI illusion, it is at least self-evident from Figure 2A that these manipulations to the assimilation effect do not produce strong differences, probably because classic assimilation is not a very strong effect to begin with.

Yet another important question also concerns assimilation: It is not clear whether assimilation affects the dimension of brightness or lightness. Brightness refers to perceived luminance, which is the perceived luminous intensity of a stimulus; in this sense, brightness also refers to the experience of luminosity and perceived illumination. Both the glare effect (phenomenal experience of luminosity) and the PI illusion (phenomenal experience of illumination) are therefore brightness effects. Lightness, instead, refers to the experience of perceived reflectance or surface color in the achromatic domainthat is, to a property that appears to be specific to a surface and should not dramatically change because of changes in illumination over time and/or space.

Given such definitions, to which category does assimilation belong? Agostini and Galmonte (2002a), in a study devoted to Kanizsa's paradox (Kanizsa, 1980), showed that unlike brightness illusions, Musatti's assimilation displays have a contrast component to them. This quality may imply that assimilation is a hybrid effect that lies between lightness and brightness, given that illusions concerning lightness tend to be based on contrast. ${ }^{2}$ If further empirical research supports this link between assimilation and contrast, it would represent a major difference between the PI illusion and assimilation.

In regard to the relationship between the PI illusion and assimilation, one more fact has still to be considered: The PI illusion shows a feature, directionality, that may not be a component of assimilation as traditionally conceived. In fact, looking at Musatti's displays (Figure 2A), the assimilation effect seems to work because elements are randomly placed all over the surface. In the case of the PI illusion, on the other hand, the effect works because of a coherent polarity chain among the inducers that defines the direction of the brightness differences not only in photometric, but also in spatial terms. In some sense, the configurations Bs/g-w, Bs/w-g, Gs/b-w, Gs/w-b, Ws/b-g, and Ws/g-b (see Figure 3 and Table 3) should represent a test for the hypothesis that assimilation may also show such a directionality component.
Such configurations are also characterized, however, by a high degree of multistability (they may determine different perceptual outcomes; see note 1). Hence, one may conclude that it is rather difficult, if not impossible, to test whether assimilation displays à la Musatti can show spatial directionality. In the present context, such impossibility or difficulty is precisely the major point of interest. Musatti's assimilation displays do not seem to advantage any particular organization of the inducers, and thus the spatial directionality of a brightness enhancement seems not even to be a component of the effect.

In conclusion, the arguments, demonstrations, and data presented in this article show the PI illusion to be a novel effect. The illusion appears to be related to the perception of differential ambient illumination, with observers' attention attracted to the brighter area. Despite some superficial similarities with other brightness and/or lightness effects, a different set of rules seem to govern the experience of this illumination illusion.

\section{REFERENCES}

Agostini, T., \& Galmonte, A. (1997). Luminance gradients, perceived illumination, and lightness perception. Review of Psychology, 4, 3-6.

Agostini, T., \& Galmonte, A. (2002a). Lightness or brightness? Kanizsa's paradox [ECVP 2002 abstract]. Perception, 31(Suppl.).

Agostini, T., \& Galmonte, A. (2002b). A new effect of luminance gradient on achromatic simultaneous contrast. Psychonomic Bulletin \& Review, 9, 264-269.

BEZOLD, W. VON (1876). The theory of color in its relation to art and art-industry. Boston: Prang.

Bressan, P. (2001). Explaining lightness illusions. Perception, 30, 1031-1046.

BuRnHAM, R. W. (1953). Bezold's color-mixture effect. American Journal of Psychology, 66, 377-385.

DA Pos, O., \& ZaMBIANCHI, E. (1996). Visual illusions and effects: A collection. Milan: Guerini \& Associates.

Gerbino, W., \& Kanizsa, G. (1987). Can we see constructs? In S. Petry $\&$ G. Meyer (Eds.), The perception of illusory contours (pp. 246252). New York: Springer.

Gyoba, J. (1983). Stationary phantoms: A completion effect without motion and flicker. Vision Research, 23, 205-211.

Helson, H., \& Rohles, F. H., JR. (1959). A quantitative study of reversal of classical lightness-contrast. American Journal of Psychology, 72, 530-538.

KANIZSA, G. (1955). Margini quasi-percettivi in campi con stimolazione omogenea. Rivista di Psicologia, 49, 7-30.

Kanizsa, G. (1979). Organization in vision. New York: Praeger.

Kanizsa, G. (1980). Grammatica del vedere. Bologna: Il Mulino.

Kennedy, J. M. (1976). Sun figure: An illusory diffuse contour resulting from an arrangement of dots. Perception, 5, 479-481.

Kitaoka, A., Gyoba, J., \& Kawabata, H. (1999). Photopic visual phantom illusion: Its common and unique characteristics as a completion effect. Perception, 28, 825-834.

Leonards, U., Ibanez, V., Zavagno, D., Seghier, M., \& Troscianko, T. (2003). A cortical region for luminosity perception: An fMRI study [Abstract]. Journal of Vision, 3, 425a.

Logvinenko, A. D. (1999). Lightness induction revisited. Perception, 28, 803-816.

McCourt, M. (1982). A spatial frequency dependent grating-induction effect. Vision Research, 22, 119-134.

McCourT, M. (1994). Grating induction: A new explanation for stationary phantom gratings. Vision Research, 34, 1609-1617.

Metzger, W. (1971). I fondamenti della psicologia della gestalt. Florence: Giunti-Barbera. 
Musatti, C. L. (1953). Luce e colore nei fenomeni del "contrasto simultaneo," della "costanza" e dell' "eguagliamento." Archivio di Psicologia, Neurologia e Psichiatria, 14, 544-577.

Newhall, S. M. (1942). The reversal of simultaneous brightness contrast. Journal of Experimental Psychology, 31, 393-409.

Plet, S., Ripamonti, C., \& Gerbino, W. (2000). Color proximity predicts intensive and chromatic effects in T-junction displays [Abstract]. Investigative Ophthalmology \& Visual Science, 41, B226.

Ripamonti, C., Gerbino, W., \& Plet S. (1998). Assimilation and contrast in the chromatic Munker-White's effect [Abstract]. Investigative Ophthalmology \& Visual Science, 39, B662.

Rosenbach, O. (1902). Zur Lehre von den Urtheilstäuschungen. Zeitschrift für Psychologie, 29, 434-448.

SAKURAI, K., \& Gyoba, J. (1985). Optimal occluder luminance for seeing stationary visual phantoms. Vision Research, 25, 1735-1740.

Tynan, P., \& SeKuler, R. (1975). Moving visual phantoms: A new contour completion effect. Science, 188, 951-952.

VAN TuIJL, H. F. J. M. (1975). A new visual illusion: Neonlike color spreading and complementary color induction between subjective contours. Acta Psychologica, 39, 441-445.

VARIN, D. (1971). Fenomeni di contrasto e di diffusione cromatica nell'organizzazione spaziale del campo percettivo. Rivista di Psicologia, 65, 101-108.

Wertheimer, M. (1923). Untersuchungen zur Lehre der Gestalt. Psychologische Forschung, 4, 301-350.

Zavagno, D. (1999). Some new luminance gradient effects. Perception, 28, 835-838.

Zavagno, D. (2001). Playing with luminance gradients: A phenomenological overview. Perception, 30(Suppl.), 19.
Zavagno, D., \& CAPUto, G. (2001). The glare effect and the perception of luminosity. Perception, 30, 209-222.

Zavagno, D., \& CAPUto, G. (in press). Glowing greys and surfacewhite. Perception.

Zavagno, D., \& Massironi, M. (1997). La rappresentazione della luce nelle opere d'arte grafica. Giornale Italiano di Psicologia, 24, 135-187.

\section{NOTES}

1. These patterns are "improper" for different reasons. For example, configurations like Bs/b-g and Bs/g-b are ambiguous, since the gestalt grouping principle known as "closure" (Wertheimer, 1923) is unresolved, given that corners - an important feature for the identification of polygons (Kanizsa, 1979) - are missing in those patterns. Also, these configurations may generate a third possible percept - that is, an anomalous hole through which portions of disks are seen. A pattern like $\mathrm{Wr} / \mathrm{b}-\mathrm{w}$, on the other hand, is "not so good" because it generates a somewhat weak glare impression, far from the actual strength of the illusion (Zavagno, 2001).

2. However, research by Gerbino (Gerbino \& Kanizsa, 1987; Plet, Ripamonti, \& Gerbino, 2000; Ripamonti, Gerbino, \& Plet, 1998) has shown that classical contrast effects, when studied with chromatic stimuli, appear to have an assimilation component to them.

(Manuscript received March 21, 2003; revision accepted for publication April 19, 2004.) 